\title{
Automated data reduction workflows for astronomy
}

\section{The ESO Reflex environment}

\author{
W. Freudling, M. Romaniello, D. M. Bramich, P. Ballester, V. Forchi, C. E. García-Dabló, S. Moehler, and M. J. Neeser
}

\author{
European Southern Observatory, Karl-Schwarzschild-Str. 2, 85748 Garching, Germany
}

e-mail: wfreudli@eso.org

Received 16 August 2013 / Accepted 14 October 2013

\begin{abstract}
Context. Data from complex modern astronomical instruments often consist of a large number of different science and calibration files, and their reduction requires a variety of software tools. The execution chain of the tools represents a complex workflow that needs to be tuned and supervised, often by individual researchers that are not necessarily experts for any specific instrument.

Aims. The efficiency of data reduction can be improved by using automatic workflows to organise data and execute a sequence of data reduction steps. To realize such efficiency gains, we designed a system that allows intuitive representation, execution and modification of the data reduction workflow, and has facilities for inspection and interaction with the data.

Methods. The European Southern Observatory (ESO) has developed Reflex, an environment to automate data reduction workflows. Reflex is implemented as a package of customized components for the Kepler workflow engine. Kepler provides the graphical user interface to create an executable flowchart-like representation of the data reduction process. Key features of Reflex are a rule-based data organiser, infrastructure to re-use results, thorough book-keeping, data progeny tracking, interactive user interfaces, and a novel concept to exploit information created during data organisation for the workflow execution.

Results. Automated workflows can greatly increase the efficiency of astronomical data reduction. In Reflex, workflows can be run noninteractively as a first step. Subsequent optimization can then be carried out while transparently re-using all unchanged intermediate products. We found that such workflows enable the reduction of complex data by non-expert users and minimizes mistakes due to book-keeping errors.

Conclusions. Reflex includes novel concepts to increase the efficiency of astronomical data processing. While Reflex is a specific implementation of astronomical scientific workflows within the Kepler workflow engine, the overall design choices and methods can also be applied to other environments for running automated science workflows.
\end{abstract}

Key words. methods: data analysis - astronomical databases: miscellaneous - techniques: miscellaneous - virtual observatory tools

\section{Introduction}

Astronomical observations produce data streams that record the signal of targets and carry associated metadata that include observational parameters and a host of associated information. Apart from the intended signal, such raw data include signatures of the atmosphere and the instrument, as well as noise from various sources. Before any scientific analysis of the data, a process called "data reduction" is used to remove the instrumental signature and contaminant sources and, for ground based observations, remove atmospheric effects. Only then, can the signal of the target source be extracted. In general, data reduction also includes a noise model and error propagation calculations to estimate uncertainties in the extracted signal.

In recent years, astronomical data reduction and analysis has become increasingly complex. The data from modern instruments can now comprise dozens of different data types that include both science and calibration data. For example, the reduction of data from ESO's X-shooter instrument uses almost 100 different data types for its three simultaneously working arms. The reduction of such data in general includes a large number of complex high-level algorithms and methods. The data types and methods are interdependent in a complex web of relations. It is therefore increasingly difficult for an individual researcher to understand, execute and optimize a data reduction cascade for complex instruments. This situation has led to the appearance of specialized, highly integrated data reduction pipelines that are written by specialists and can reduce data without supervision (e.g. Biretta et al. 1994; Jung et al. 2004; Tucker et al. 2006; Schmithuesen et al. 2007; Cavanagh et al. 2008; Tsapras et al. 2009). For efficient large scale data reduction, such pipelines often run in custom-made environments. For example, ESO employs a system for quality control that automatically associates calibration data and processes them as soon as they arrive from the telescopes. The results are then stored in its data archive. Other examples of such event driven data reduction environments are NOAO's High-Performance Pipeline (Scott et al. 2007), STScI's OPUS system (Rose et al. 1995), and the Astro-WISE pipeline (McFarland et al. 2013).

Automatic pipelines work best for data from long-term projects that use stable instrumentation, aim for a well-defined set of similar targets observed at similar signal-to-noise ratio, and in situations where the impact of ambient conditions is relatively small and highly predictable. These conditions are often met, for example, in space-based telescopes. However, the situation is often different for the reduction of data from groundbased observatories. The reasons for this include the complexity of general purpose instruments that are now routinely employed, the rapid upgrade pace necessary to exploit advances in technology and science goals, and the variety of effects imposed by varying atmospheric conditions. In many cases, supervision and interaction with the data reduction process is, therefore, still 
essential to obtain sufficiently high quality results even from fairly routine observations.

The general concept of astronomical data reduction that does not employ a fully integrated pipeline has not substantially changed in the past decades. Researchers organise their data, and use a mixture of general purpose and highly specialized tools, inspecting the results of each step. Such tools are available in environments such as MIDAS (Banse et al. 1983), IRAF (Tody 1993) and $\mathrm{IDL}^{1}$, or as stand-alone programs. What has changed is the number, complexity and interdependence of steps needed to accomplish the data reduction. In this situation, the efficiency of the data reduction process can be vastly improved by automating the previously manual workflow of organising data, running individual steps, and transferring results to subsequent steps, while still using the same routines to carry out individual reduction steps.

The most commonly used approach to automate a data reduction workflow by individual researchers is to employ a scripting language such as Python (e.g. Nastasi et al. 2013). This approach works well with a relatively small number of reduction steps, and in situations where the data organisation and book-keeping are fairly simple. In more complex situations, such scripts are themselves complex programs that cannot easily be modified.

In this paper, we describe the usage of a general workflow engine to automate the data reduction workflow for astronomical observations. While this approach is relatively new for the field of astronomy (e.g. Ballester et al. 2011; Schaaff et al. 2012), it has been widely used in other fields of science including biology and chemistry (Abhishek \& Sekharb 2007), meteorology (Barseghian et al. 2009) and economics (Ludäscher et al. 2005). For that reason, we discuss in detail the methods and functionalities that are necessary to use such a system for astronomical data reduction, and present ESO's new “Recipe flexible execution workbench" (Reflex) environment as a specific implementation of such a system.

The structure of the paper is as follows. In Sect. 2, we describe the main principles and architecture of our design independent of a particular implementation. In Sect. 3, we discuss how these principles can be implemented in a specific workflow application, using our Reflex implementation as an example. Finally, in Sect. 4 we conclude with a discussion of the impact of performing data reduction in this way.

\section{Architecture of astronomy data reduction workflows}

\subsection{Data organisation}

Astronomical data consist of collections of files that include both the recorded signal from extraterrestrial sources, and metadata such as instrumental, ambient, and atmospheric data. Such a collection of files is the raw output from one or several observing runs, and consists of "science files" that contain the primary science observations to be analyzed.

In addition, it might include files that are not directly related to the current observations, such as calibration files that are routinely collected for a given instrument. Hereafter, we refer to the input files for the data processing as "raw files", as opposed to files that are created during the data processing and that we will

\footnotetext{
1 IDL is a trademark of Research Systems Inc., registered in the United States.
}

refer to as "products". We use the term "calibration file" for any raw file that is not a science file.

In order to discuss data reduction in general terms, we introduce the following terminology. The goal of data reduction is to process sets of files, which we refer to as the targets of a data reduction workflow. The result of this processing is to create a target product. In most cases, the targets of a data reduction workflow will be the science files, and the target product is then the science data product to be used for scientific analysis. The target files can be naturally grouped into sets that are reduced together. Such a group of target files, together with other files needed to process them, is referred to as a data set. A data set is "complete" when it contains all necessary files to reduce the targets, and "incomplete" if some of those files are missing.

"Data organisation" is the process of selecting data sets from a larger collection of files, and recording information on the type of files and the reasons for selecting them. This larger collection of files might be the result of a pre-selection process that assures that low quality or defective data are not considered at this stage. Organising data is a complex and time-intensive procedure that is typically among the first tasks of a data reduction workflow (e.g. Scodeggio et al. 2005). Hereafter, we will refer to the whole data reduction workflow including data organisation simply as a "workflow", whereas we will use the term "data processing workflow" for the processing of data that follows the data organisation.

The first step in data organisation is to classify files, i.e. to determine the data content of each file from its metadata. The goal of classification is to assign a category to each file. An example of such a category is "flatfield for filter I". The next step is to identify the targets, and group them into data sets that are incomplete at this stage. Subsequently, calibrations are added to the data sets. Calibration files for each data set are selected by analysing the metadata of the targets and that of other available files that potentially qualify for inclusion in a data set.

This cascade of selection criteria naturally maps into a data graph as illustrated in Fig. 1. The links between elements of the graph show the flow of metadata that originates from the raw files. The graph is directed, i.e. links between elements have a direction to distinguish between incoming and outgoing information. The nodes of the graph define necessary procedural steps in the assembly of a data set, and we refer to them as actions. The targets of the workflow connect directly to the root node (action 1) that is therefore called the target action.

Each action has several incoming files connected to it. Some of those are used to define selection properties of other input files to that action. For example, an action might specify to select flat field images that use the same filter as the science image. We use the notation that the files that are used to define properties of other files, in our example the science files, are the trigger for that action, and their links are shown as solid lines in Fig. 1. The trigger of the target action are the targets of the workflow.

All actions other than the target action have one or several outgoing links that connect them to subsequent actions. These outgoing links pass on metadata that are extracted from the input files to the next actor. They are therefore called products of an action. These products do not necessarily correspond to actual physical products produced during data reduction, and the actual physical products created during data reduction do not necessarily appear in the data organisation graph. Instead, the products in the data organisation graph are used as a logical scheme to define the selection of data. For example, for the purpose of data organisation, it is not necessary to define a target product, even when the data processing workflow creates one. This is because 
Dataset

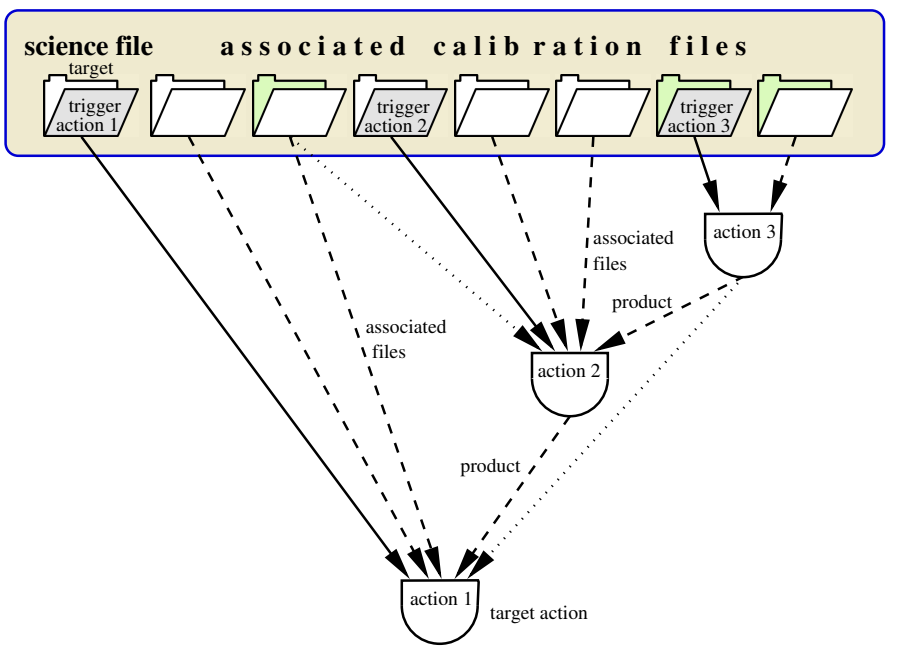

Fig. 1. Example of a simple data set and its organisation. The data set contains all files necessary to produce the science data product of the workflow. This includes the science and associated calibration files. These files are organised using a set of actions that are shown as shieldshaped symbols. The target files are directly connected to the target action that is the root of the graph. Files that are connected to an action with a solid line are the trigger for that action. Properties of the triggers are used to select associated files for an action. The associated files are connected to an action with dashed or dotted lines. To highlight files that are connected to more than one action, a dashed line is used for one of these connections, and a dotted line for the other one. The purpose of a file is the connection between the file and the target action. Symbols with tinted background indicate files that have multiple purposes, i.e. there are multiple paths from the file to the target action.

the nature and properties of the target product have no impact on the data selection.

Each raw file is the origin of at least one path along the direction of the links that lead to the target action. This reflects the fact that data sets only include raw files that are needed to process the targets of the workflow. A path runs either directly from the files to the target action, or passes through other actions on its way. We refer to such a path as one of the purposes of a file. The purposes of a file are important information for the data processing (see Sect. 2.3).

In Fig. 2, we show the data graph for a specific example with the same symbols used in Fig. 1. The simple example is an image that needs a bias frame, a flatfield and a dark frame for its processing. The flatfield needs to be taken with the same optical filter as the science frame, whereas the dark frame needs to be taken with the same exposure time as the science frame. Therefore, flatfields and dark frames with these properties must be identified among available files, and one of each must be selected according to criteria such as the closeness of the time of observation to that of the science frame. After this step, more calibration files need to be added to the data set that are used to reduce the calibration files. The selection criteria for those files depend on properties of calibration files instead of the targets of the workflow. In the current example, the flatfield itself needs a dark frame for its processing, and this dark frame needs to match the exposure time of the flatfield, not that of the science frame. The science frame, flatfield frame and dark frame in turn might all require their own bias frame for reduction. The actions in this case are given specific labels, namely "proc_dark", "proc_flat" and "proc_image". Note that the action "proc_dark" is shown
Dataset

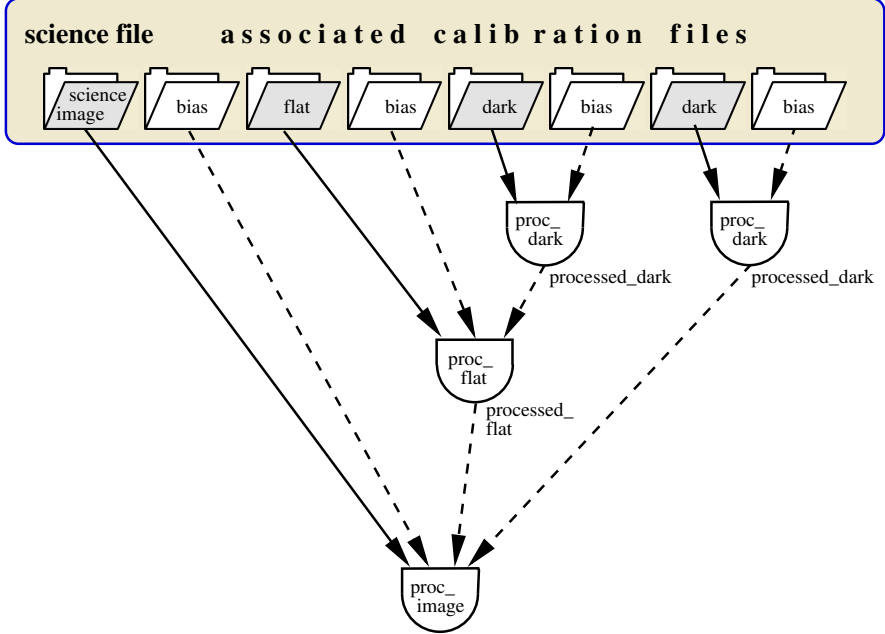

Fig. 2. Data graph for a data set to process images as described in the text. The symbols used are the same as in Fig. 1. In the case shown here, each file has a unique purpose, and therefore no dotted lines are used. The action "proc_dark" is used to select different darks for the flat frame and the science image. Therefore, it appears twice in the graph.

twice, reflecting the fact that it is used twice, once to select darks for the flatfield, and a second time to select darks for the image.

We note that the topology of the graph might differ between data sets even for the same kind of data. For example, in one data set the input dark frames for the science and flat frames might be identical, in another one they might differ. The task of data organisation is to create such a graph for each individual data set.

\subsection{Design of data organisation and data processing systems}

The data organisation discussed in Sect. 2.1 and the data processing that follows the data organisation both describe relations among different categories of data. These relations are interdependent, in the sense that a change in the selection of data might require some change in the data processing, and vice versa. The question therefore arises as to whether the best architecture is to derive these relations from a common source, or whether the information recorded in these relations is sufficiently different to warrant their independent implementation.

In general, a specific selection of data does not uniquely specify the data processing sequence. Very different data processing workflows can be constructed to use a given selection of data. Only the most basic data processing follows the data organisation process one-to-one, but this case is rarely used in practice. The data processing part of the workflows are, in general, more complex than the data organisation, and are also more frequently subject to change and optimization during the data reduction process. The purpose of a file records an aspect of the selection criteria used to include this file in a data set. It is up to the workflow design to decide how this information is used.

For example, a category of files (such as a flatfield) might be selected to match the date of the science frames and is, therefore, assigned a corresponding purpose. This does not necessarily mean that these flatfields are exclusively used to flatfield the science frames, but the data processing workflow might also use them to flatfield standard star flux calibration data. For 
spectroscopy, it is not always clear whether the best flatfields for the flux calibrator are those that are taken close in time to the target spectrum or those taken close in time to the flux calibrator. This decision depends on a complex set of circumstances. A workflow might include conditional and/or interactive parts to help the user make that decision.

Another difference between data organisation and data processing is that, while some steps in the data processing are closely related to a specific selection of data, others are completely independent of it. For example, a step that only modifies intermediate products has no impact on the data selection or organisation. Steps that make small adjustments to intermediate products are often added or removed during data reduction. Any system that mixes the data selection and data processing workflows is then, necessarily, much more complex than either of the two components individually. One design goal for a workflow system is to make modification of the data reduction as simple as possible. This is helped by clearly separating the data organisation from the data reduction steps.

We therefore advocate a design that not only separates the implementation of the two steps, but also uses a different methodology to define the two tasks. Each of them should be geared towards the specific needs of each step. The data organisation is usually closely related to the instrument properties, the observing strategy and the calibration plan. The strategy for data organisation therefore rarely changes after the observations have taken place. Interactivity in that part will create overheads that do not outweigh the expected benefits. On the other hand, the data processing is, in general, highly interactive and experimental, and the final strategy is rarely known at the time of observation. The best values for data reduction parameters and even the chosen strategy might depend on the properties of individual data sets.

An efficient way to implement a data organisation is, therefore, a rule-based system that can accommodate complex, instrument-specific rules and can be run to organise either locally stored data or data extracted from an archive repository using pre-defined rules. The syntax of the rules must be able to describe the method of creating data graphs such as the ones discussed in Sect. 2.1. Such data organisation is particularly efficient if it is carried out by raw-data archives that have any potentially useful calibration file available for retrieval. For example, ESO offers an archive service "calselector"'2 that selects, organises and provides access to data in a manner similar to the one described above.

In contrast, data processing after the data organisation benefits from interactive, graphical and dynamic elements. An efficient way to provide this is to use a workflow application that allows the implementation of workflows that can be easily modified for experimentation and optimization during data processing. It is important that these interactive features can be turned off once a workflow has been tuned and optimized, in order to allow time-intensive processing to be carried out in a batch mode.

\subsection{Functionalities of a rule-based data organiser}

A software program that uses rules to organise data as advocated above can produce the data graph discussed in Sect. 2.1 by a set of steps shown in the flow chart of Fig. 3. The output of the data organisation is a list of data sets. A data set is marked as "complete" if there are files that satisfy the criteria used in steps shown in shaded boxes in Fig. 3. It is marked as "incomplete" if any one

\footnotetext{
2 http://www.eso.org/sci/archive/calselectorInfo.html
}

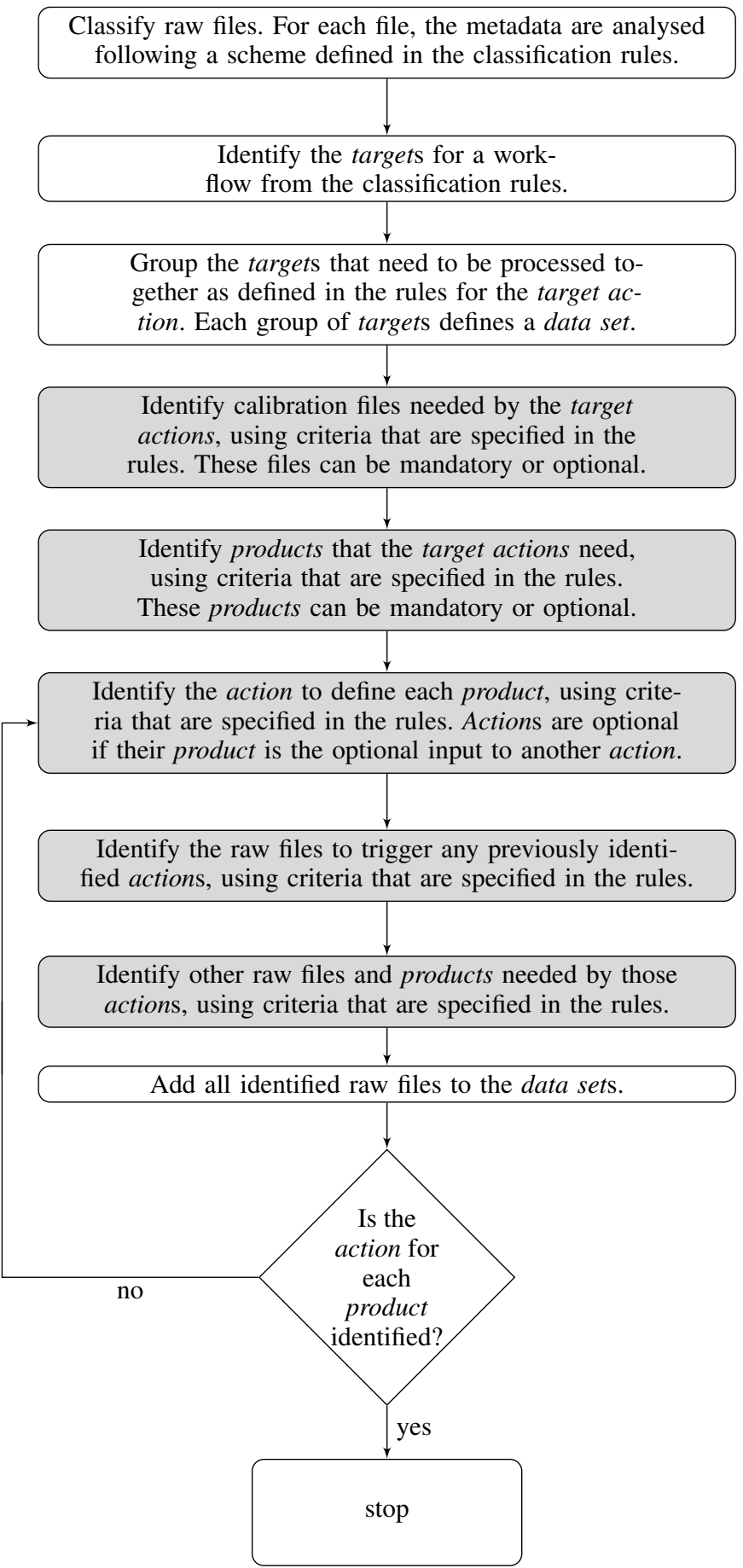

Fig. 3. High-level flow chart of a data organiser. If any step in a shaded box fails for any given data set, then this data set is marked as "incomplete".

of those criteria are not satisfied by any existing file. Each file in the output data sets is described by the file name, the category of the file as defined in the rules, and the purpose of the file. The purpose of the file is recorded as the concatenation of the names of the actions that link the file to the target action. In the example discussed in Sect. 2.1, the flatfield is selected based on properties (in this case the filter) of the science frame. The selection rules are defined in the action called "proc_science". Then the bias frame is selected for this flatfield based on properties of the flatfield (e.g. observing date or read-out mode). This selection is defined in the action "proc_flat". The purpose of this bias frame, 
as well as the flatfield, is then "proc_science:proc_flat", while the bias frame that matches the properties of the science frame, as well as the science frames themselves, have the purpose "proc_science". The other biases in this example have the purpose "proc_science:proc_flat:proc_dark" and "proc_dark". The different biases have different purposes so that the workflow can process them separately. A given file might have several different purposes if it is selected multiple times by the rules (see Fig. 1). An example of this is when the same bias frame matches the selection rules for both the flatfield and the science frames.

\subsection{Data processing workflows}

There are different ways to carry out the task of reducing astronomical data, even when the applications used for individual reduction steps are fixed. One approach is to sort data by category, and process each category in sequence. For example, one might start by processing all the bias frames for all data sets as the very first step, then proceed to subtract combined biases from all relevant data, and continue with, for example, producing flatfields.

A different approach is to fully process a single data set, performing all necessary steps to see the final result for the first data set in the shortest possible time. Each intermediate product, such as a combined bias, is produced only when it is needed.

The former approach has the advantage that it simplifies book-keeping, in that the only necessary initial sorting is by file type. Operations of the same kind are all performed together. The parameters for every task are optimized by repeatedly inspecting results. Once a good set of parameters is found, it is applied to all files of the same kind. This approach is efficient in the sense that identical operations are carried out only once, while the effort for bookkeeping is minimal. It is, therefore, often used when workflows are manually executed by scientists that call individual steps in sequence and book-keeping is carried out ad-hoc without software tools.

The advantage of the latter approach is that it allows for easier inspection of the impact of any change in parameters or procedures on the quality of the final target product of a workflow. This is particularly important when data reduction strategies are still experimental and being tested. This approach also delivers the results faster in that it only executes the steps that are needed for a given data set and thereby more quickly produces the target product for the first data set.

The advantages of both of these approaches can be combined with the following design. As in the second approach, data are processed one data set at a time. Data reduction steps that need to be executed several times with different input files from the same data set are carried out in succession. For example, the step to combine bias frames is executed for the biases to be applied to the science frame, and immediately afterwards the bias frames to debias the flatflields are processed, and so on. The inputs and outputs of each individual data reduction step are stored in a database for re-use later. Whenever a reduction step is called, this database is checked for previous calls to the reduction step with the same input files and parameters. If such a previous call exists, then the reduction step is not executed and instead the previous results are re-used. We call the feature to re-use products created by previous executions of a procedure the "lazy mode". There might be cases when such a re-use of products is not desired. A lazy mode should, therefore, always be an option of each individual step in a workflow. An example for the efficiency gain from using this mode is a set of combined biases that are used by the science and flatfield frames of a data set, and in addition

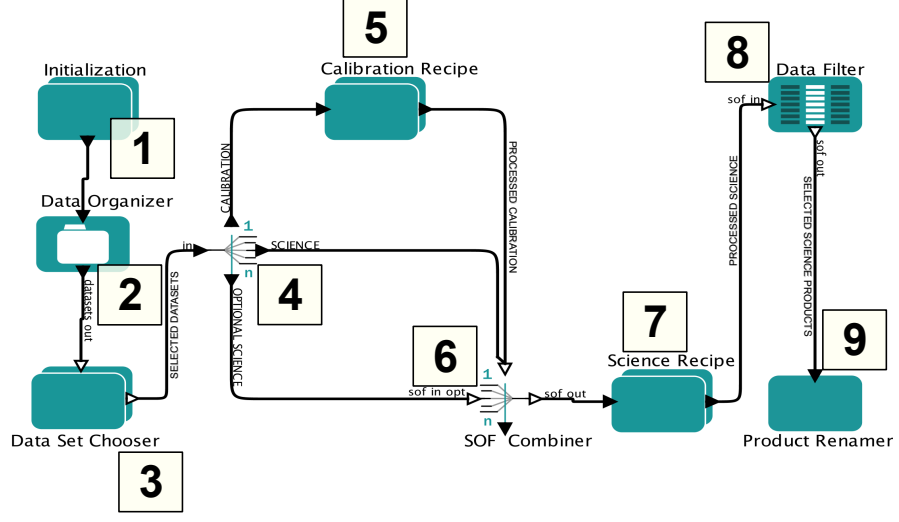

Fig. 4. Example of a basic Reflex workflow. The figure uses the graphical elements of a Kepler workflow (Sect. 3). The lines indicate the flow of files and are labelled by their contents. The "optional science" files are files that are used to process the science data, but the processing can proceed even if they are not available (see Sect. 3.3.3). The workflow includes two data processing steps, one for calibration and one for science processing (labelled [5 and 7, respectively). The elements of the workflow are: an initialization 10 that sends the input directories to the data organiser, the data organiser 2, a data set chooser 3 that allows interactive selection of a data set, the file router 4 that directs different categories of files to their destinations, a SOFCombiner 6 that bundles the input for the science step, and a data filter and product renamer ( 8 and 9 , respectively) that organise the output products from the workflow.

by the calibration frame of another data set. The combination of biases is carried out only once, and is used in three different places. One advantage of our approach is that it is as efficient as the first of the above approaches, but produces the science results quickly and provides the user experience of the second approach. Another advantage is that subsequent runs of the workflow can use this database of intermediate products to redo the reduction with changed parameters in a very efficient manner. If a parameter or input file for any step changes, then the result for this step will change. The change in one of the intermediate products might require the re-execution of some but not all of the subsequent steps. The database can be used to automatically identify products that can be re-used from previous runs, and the steps that need to be repeated.

The implementation of this workflow design requires three levels of grouping of data. A schematic diagram of such a workflow is shown in Fig. 4. The highest level of grouping are the data sets, as discussed in Sect. 2.1. This task is carried out by a data organiser (step 2 in Fig. 4). Subsequently, the files in each data set are sorted by category and directed to the reduction steps that need this particular category of files, a step that is performed by a file router (step 4). This is the level that describes the data reduction strategy and is shown in the design of a workflow. Each reduction step might be called repeatedly with different input files. For that purpose, a third level of grouping is needed to group files that are processed together with separate calls of the reduction step. This is part of the functionality of the reductions steps 5 and 7 .

\section{Implementation}

While the principles discussed in this paper do not depend on a specific software implementation, it is useful to discuss them in the context of, and with the terminology used, in a specific environment. Several software environments to design and execute workflows exist (e.g. Curcin \& Ghanem 2008). For the Reflex 


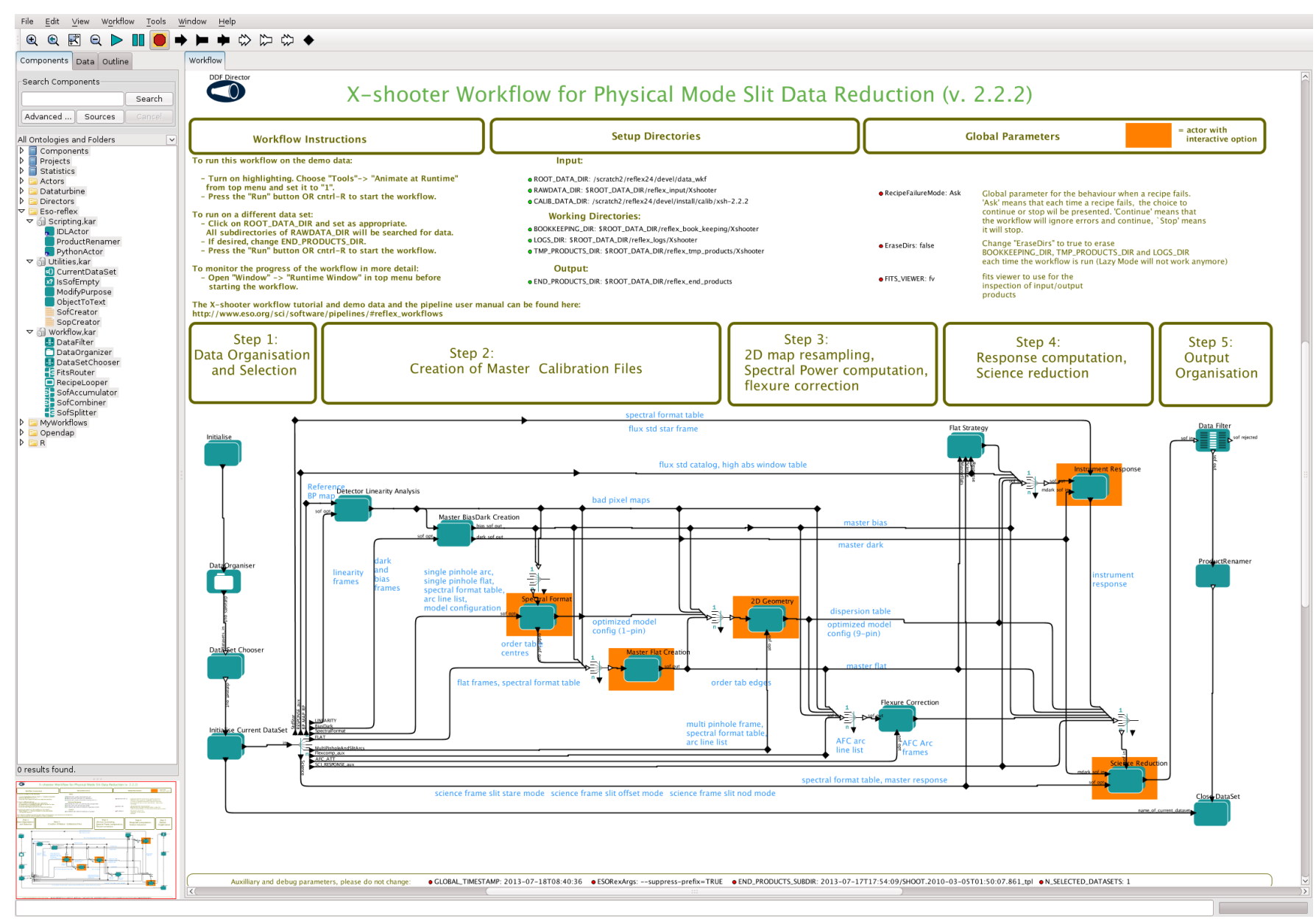

Fig. 5. Kepler user interface loaded with the Reflex workflow for ESO's X-shooter instrument. The top section defines the input directories and user preferences. It is usually sufficient to specify the raw data directory to run the workflow on a new data set. The execution of a workflow is started with the run button in the top left panel. The workflow includes 8 recipe executers that run the recipes necessary to reduce X-shooter data. Actors with an orange background include interactive steps that can display the result of the recipe, and allow for the optimization recipe settings. The workflow includes an specially implemented actor called "Flat Strategy" that is specific to the X-shooter workflow. This actor allows the user to select a flatfielding strategy. Depending on the chosen strategy, files will be routed differently.

project, we evaluated and partially implemented some of the concepts discussed in this paper in several open source workflow engines. In the end, we decided to use the Kepler workflow application (Altintas et al. 2004) to implement Reflex, because of its large suite of available components, and its robust support for conditional branching, looping, and progress monitoring. In this section, we introduce the terminology and summarize the most important features of Kepler. For more details, see the Kepler User Manual ${ }^{3}$.

\subsection{The Kepler workflow engine}

A workflow application is a software system designed to run sequences of stand-alone programs, where the programs depend on the results of each other. Components of a workflow are representations of these programs, as well as elements that manage the results and communication between them. In Kepler, components of the workflow are called "actors". In the graphical interface, actors are represented by green boxes (see Fig. 5). Actors have named input and output "ports" to communicate with

\footnotetext{
3 https://code.kepler-project.org/code/kepler-docs/ trunk/outreach/documentation/shipping/2 .4/UserManual. $\operatorname{pdf}$
}

each other. The communication is implemented by exchanging objects called "tokens" that travel along connections between the output port of one actor to the input port of another actor. These connections are called "relations" and are represented by lines. Output ports emit tokens after the execution of an actor is finished, and input ports consume tokens when execution of the actor starts. The availability of tokens is a crucial factor in determining the sequence of triggering the actors.

The relations between actors themselves do not define the temporal sequence of the execution of actors. A scheduler is required to trigger the execution of each actor. A scheduler in a Kepler workflow is called a "director". The terminology of Kepler follows the metaphor of film making, where a director instructs actors that carry out their parts. Reflex uses the "Dynamic Data Flow" (DDF) director that allows the workflow execution to depend on the results of actors and supports looping and iterating. The basic algorithm used by the DDF director is to repeatedly scan all actors and identify those that can be executed because all of the necessary input is available. It then selects one of the actors for execution based on minimizing unused tokens and memory. The details are extensively discussed in Zhou (2004). It should be noted that an actor of a workflow can itself 
be a sub-workflow. Such "composite actors" might include their own directors.

The Kepler workflow application provides a graphical interface to create, edit and execute workflows. A large number of general purpose actors are bundled with the environment. There are several ways to monitor the progress of a workflow, pause it, or stop it.

\subsection{The reflex environment}

We have produced the software package Reflex to implement the design discussed in this paper using Kepler workflows. Reflex consists of a collection of actors that support the execution of astronomical applications. A shared characteristic of commonly used astronomical applications is that they read data and metadata from FITS files, are configurable with parameters, and produce output FITS files called products. Reflex supports any application of this kind that can be started from a command line. Hereafter, we refer to such applications as "recipes". The primary task of Reflex is to route the necessary input files to the recipes. This includes both files in a data set and files created during execution of the data processing workflow. In addition, Reflex is able to create and send lists of parameters to recipes.

To achieve these tasks, Reflex uses two kinds of objects called "set of files" (hereafter SOF) and "set of parameters" (hereafter SOP). These objects are used as tokens in a workflow. A SOF contains a list of files. The record for each file consists of the file name, the checksum, the category and a list of purposes for that file. A SOP contains a list of parameters, and the record for each parameter consists of its name and value. Reflex actors use and process these objects.

The construction of an input SOF, to be fed to a recipe, needs to consider the category and the purpose of a file. For every file in a data set, these file properties are determined during file organisation according to the pre-defined rules. For products, these properties have to be determined during the execution of the workflow. It is important to note that these two file properties are handled differently. Every recipe needs to be aware of the file category of its input files. For example, a recipe that combines flatfields might use dome flat exposures and bias frames as input files, and these files need to be identified to the recipe. The mechanism to identify files to the recipes is different in different environments. For example, IRAF uses different input parameters for different file types, whereas ESO's CPL recipes (McKay et al. 2004) use text files with file tags to identify the file types. In both cases, Reflex uses the category to identify these file types. Reflex workflows therefore need to explicitly use the exact names known to the recipes for its categories. The data organisation rules have to generate these exact names. In contrast, recipes are oblivious to the purpose of a file. The recipe to combine flat field frames does not need to know how and where the combined flatfields will be used. Therefore, the processing of the purpose is completely handled by workflow actors.

As discussed in Sect. 2.3, a purpose is a concatenation of actions used to organise the input data. The name of an action is arbitrary and, therefore, it is never used explicitly in the workflow. Instead, Reflex uses the overriding principle that recipes receive files of the same, but arbitrary purpose. Actors compare and manipulate, but never decode the purpose. There are three standard operations on the purpose, they are called passthrough, set-to-universal and trim. The operation pass-through simply reads the purpose of a file, and passes it on without any modification. The operation set-to-universal replaces an existing purpose with a new one with the protected name universal.
The universal purpose is a wildcard that may be substituted by any other defined purpose depending on the circumstances. The operation trim modifies a purpose by removing the last action from a purpose that consists of at least two concatenated actions, and sets a purpose that consists of a single action to universal. Workflows are designed so that the input to any recipe consists of files with a single identical purpose. These operations are sufficient to design workflows that collect all necessary input files for each recipe, by selecting all files with identical purposes to be processed.

The usage of these operations can best be explained with examples. The most commonly used operation is trim. In Sect. 2.3, we already used the example of bias frames with the purpose "proc_science:proc_flat" and "proc_science", and flatfield and science frames with the purpose "proc_science". When the flatfield is to be processed, the workflow selects the file with the category "flat", and all files with identical purpose. In our example, these are the bias and flatfield files with purpose "proc_science:proc_flatfield". The output product, i.e. the processed flatfield, should be assigned the trimmed input purpose. In our case, the purpose "proc_science:proc_flat" is reduced to "proc_science". In a subsequent step, a science recipe collects all the files with purpose "proc_science" for the input. This will include the processed flatfield file, the bias frame selected to match the properties of the science frame, and the science frame itself.

The operation pass-through is used for recipes that only use intermediate products as inputs. If such a recipe is needed in the chain (e.g. to smooth the flatfields in the above example), this recipe should pass-through the purpose of its input file to the product file, so that the purpose of the smoothed flatfields is still "proc_science". In general, any recipe that has no impact on the data selection should pass-through the purpose of the input files.

Finally, the set-to-universal operation can be used for files with a unique category that can be processed independently of their usage in the workflow. For example, a bad pixel map that is used by many different recipes in a workflow can be given the purpose "universal" to simplify the workflow design.

These three operations allow an efficient and elegant assembly of input files for recipes. Different operations might be necessary under special circumstances and a flexible system will allow these to be implemented. An important design principle for any operation on the purpose of a file is that it should never explicitly use the name of the purpose. The names assigned by the rules are arbitrary, and a change of those names should not impact the workflow execution.

\subsection{Reflex actors}

In order to implement these principles, Reflex provides 17 essential actors. A complete list of actors is given in Appendix A and are described in detail by Forchi (2012). The actors can be grouped into the data organiser, actors to process and direct tokens, actors to execute data reduction recipes written in one of several supported languages, and actors that provide interactive steps in a workflow. In this section, we discuss these features and options to illustrate how the principles laid out above can be implemented in concrete software modules.

\subsubsection{Data organiser and rule syntax}

For Reflex, we opted to implement a program DataOrganiser that carries out the organisation of local data fully automatically using a set of user-supplied, human-readable rules. The input of 
Table 1. OCA rules syntax.

\begin{tabular}{|c|c|}
\hline Rule type & Syntax \\
\hline Classification & if condition then $\{$ REFLEX.CATG = "category"; REFLEX.TARGET $=“[T, F] "\}$ \\
\hline Organisation & $\begin{array}{l}\text { minRet }=i \\
\text { select execute(actionname) from inputFiles where conditions [group by keyword-list] }\end{array}$ \\
\hline Association & $\begin{array}{l}\text { action actionname }\{ \\
\text { minRet }=i \text {; maxRet }=j ; \\
\text { select files as label from inputFiles where conditions } \\
\text { [closest by keyword] } ; \\
\text { product label }\{\text { REFLEX.CATG = category }\} ;\end{array}$ \\
\hline
\end{tabular}

Notes. The table lists a simplified version of the OCA rules syntax appropriate for data organisation in Reflex workflows. The conditions define categories of FITS files by their header keywords and may include logical and arithmetic expressions. The labels in the association rule are used for logging purposes and are usually set to the category of the file defined in the rule.

the DataOrganiser is a set of FITS files and the classification rules; the output is a collection of data sets. The rules are based on the principles discussed above. It should be re-iterated that, while definitions of actions could be used to define a data structure in sufficient detail to allow automatic derivation of a simple data processing workflow, this is not the approach that we adopt here. Instead, the rules are used only to organise the data, while the workflow to reduce the data is not constrained to using the selected data in any particular manner.

The DataOrganiser is the first actor after the initialization in any Reflex workflow. It organises the input FITS files according to the workflow-specific input rules, and the output are data sets that are either marked complete or incomplete (see Sect. 2.1). The execution of a Reflex workflow is triggered by sending an input token to the DataOrganiser actor.

The DataOrganiser recognizes rules that use the syntax of a special language called OCA. The OCA language has been developed at ESO (Zampieri et al. 2006) and is designed to describe the Organisation, Classification, and Association of FITS files based on their FITS header keywords (Wells et al. 1981). OCA is used for multiple purposes within ESO's data flow system (Peron 2012), and interpreters are embedded in a number of applications. Therefore, OCA rules to organise data are available for most instruments on ESO's telescopes. The details of the language are described in Zampieri \& Forchi (2012). The language has all of the features needed to define rules for data organisation. Here, we summarize a subset of the OCA language that is useful for the data organisation discussed in this paper.

The OCA language recognizes three types of rules. They are:

1. Classification rules. Classification rules define file categories based on any logical combination of conditions on FITS keywords. The syntax of the classification rules is given in row 1 of Table 1 . The classification defines the keyword REFLEX.CATG as the category of the file, and this keyword can be used like any other FITS keyword in the header by other rules. A simple example for the usage of a classification rule is to assign to a file the category "bias" if the header keyword "EXPTIME" is set to the value " 0 ". The classification rules also define whether a set of files is the target of the workflow or not.

2. Organisation rules. Organisation rules define actions and the groups of files that trigger them. The rules define a name for each action so that it can be referred to by other rules. The syntax of the organisation rules is given in row 2 of Table 1. The rules include an optional specification of the minimum number of files needed to trigger the action. This minimum number is used to determine whether a data set is complete or not. There is no maximum number because there are no defined criteria to select among files that match the condition. A simple example is to group at least 3 dome flat frames by filter, and trigger an action called "proc_flat" that combines flatfields.

3. Association rules. Association rules define "associated files", i.e. input files and products that are needed by an action in addition to the trigger. The syntax of the association rules is given in row 3 of Table 1 . There is an unlimited number of "select" statements that define conditions to select files. In addition to the conditions, a "closest by" statement can be used to select those files that have a value for a given keyword that is as close as possible to that of the trigger. If there is no "closest by" statement, then the time of observation will be used to select among several files that satisfy the conditions. Each select statement can be preceded by an optional specification of the minimum and maximum number of files needed for each category. This mechanism allows to define optional input files that are not essential for a workflow but that will be used if present. The association rules also define names for categories of products that can be referred to by other rules. A simple example is that the proc_flat action needs a combined bias frame and produces a product "MasterFlat".

These rules are sufficient to describe the data graphs discussed in Sect. 2.1 and shown in Figs. 1 and 2. In Table 2, we show as a specific example the rules that describe the data organisation for an image that needs a flatfield and a dark frame for its processing, as discussed in Sect. 2.1 and shown in Fig. 2. The first block classifies available files as "science_image", "flat" or "dark" based on the header keyword "TYPE", and as "bias" based on the fact that the value of the header keyword "EXPTIME" is 0 . The next three "select" statements define the three actions "proc_dark", "proc_flat" and "proc_image", and their triggers "flat", "dark" and "science_image". What follows are the association rules that specify that the action "proc_dark" needs a bias as input and outputs a product called "processed_dark", the $a c$ tion "proc_flat" needs this "processed_dark" and a bias, and outputs a "processed_flat". Finally, the action "proc_image" needs a "bias", the "processed_dark" and the "processed_flat". The association rules also specify that darks are selected to match the exposure time of the "science_image" or the "flat", and flats are selected to match the filter of the "science_image". The application 
Table 2. Simple example of OCA rules.

Classification rules

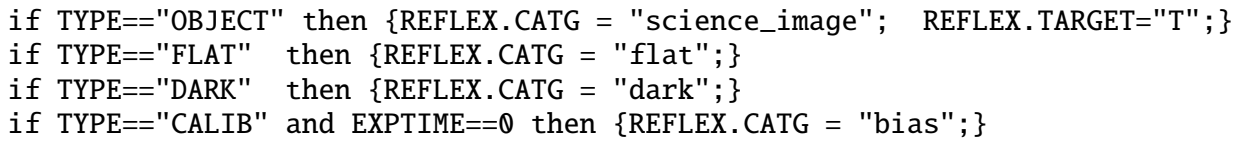

Organisation rules

select execute(proc_dark) from inputFiles where REFLEX.CATG=="dark"

select execute(proc_flat) from inputFiles where REFLEX.CATG=="flat"

select execute(proc_image) from inputFiles where REFLEX.CATG=="science_image"

Association rules

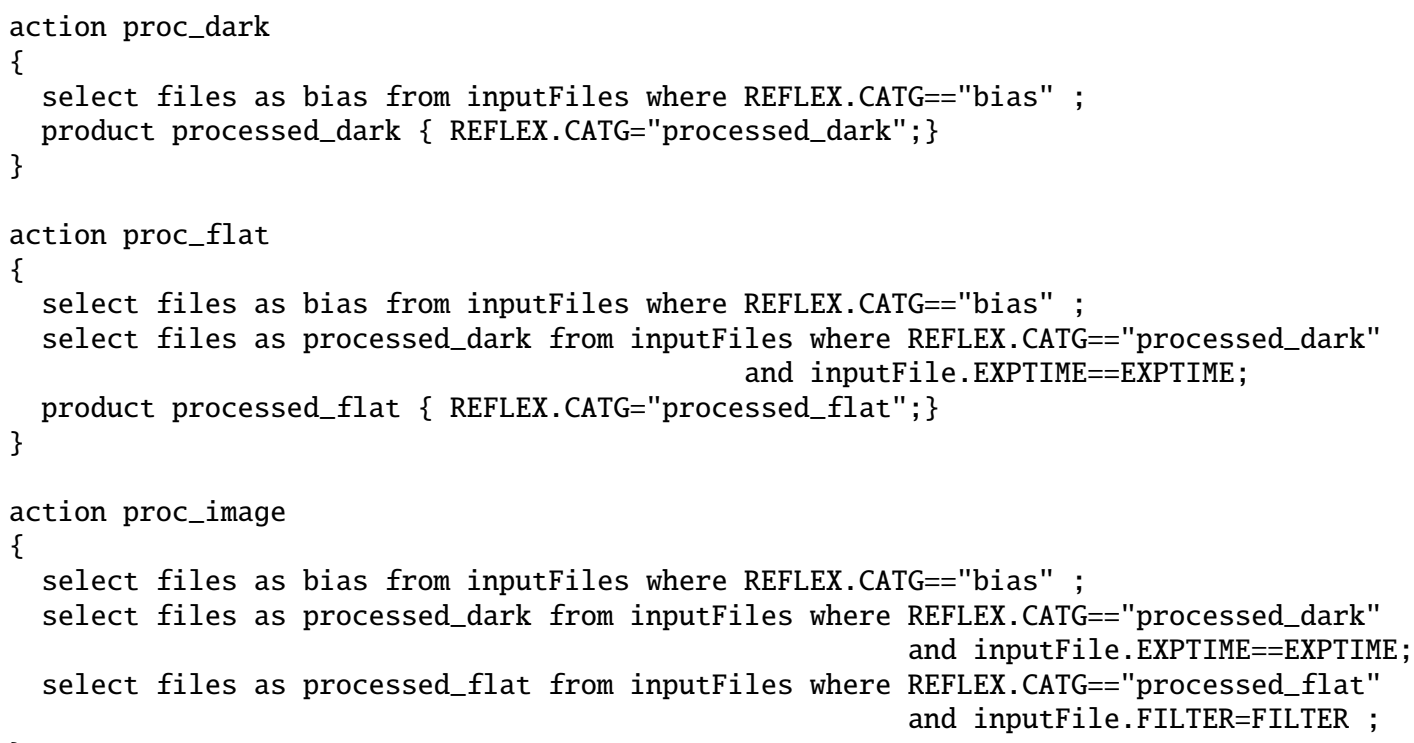

Notes. The table lists an example of a set of OCA rules that can produce the data organisation shown in Fig. 2.

of these rules can lead to a data set organized as shown in Fig. 2. However, the same rules can also lead to a data graph with different topology for a different data set. For example, if both the science image and the flat have the same exposure time, the application of the rules might select the same dark frame for both the flat and the science image. The power of the data organiser is to use such abstract rules to select optimal data sets based on the metadata of the available files.

\subsubsection{Actors for data processing}

The purpose of a data processing workflow is to execute a series of recipes. The recipes can be written in any language, but must accept the basic input, and provide the basic output information, needed to run the workflow. In particular, the recipes must accept FITS files that are categorized, and generate products as FITS files and the information to categorize them. Reflex provides three actors to execute recipes. They are called "PythonActor", "IDLActor" and "RecipeExecuter". The PythonActor is used to run Python scripts that, in turn, can call, for example, shell commands, IRAF tasks via the PyRAF interface (White \& Greenfield 2002), or MIDAS programs via the pyMIDAS interface (Hook et al. 2006). The IDLActor is used to run IDL programs, and the RecipeExecuter executes CPL recipes. The basic function of all three actors is to filter and send the files of the input SOF to the recipe, and create and emit the output SOF with the products of the recipe. The purpose of the product files is constructed from those of the input files using one of the standard operations described in Sect. 3.2. All CPL recipes can be queried to report their input and output in a well-defined format. This feature is used by Reflex to automatically generate parameter lists and ports. For Python and IDL, simple interfaces are provided that can be added to any program.

\subsubsection{Actors for file routing}

The top level task when designing a data processing workflow is to decide on the cascade of file processing, i.e. the routing of files by category. In Reflex, users of a workflow are presented with a visual diagram that shows the directional flow of files with different categories to the corresponding data processing actors (see Fig. 4). The data sets created by the DataOrganiser are SOFs that contain a full set of file categories. An actor is needed to direct the different categories of files in a data set to the respective data processing actors. In Reflex, this actor is called the FitsRouter. It takes a single data set SOF as input, and 
creates SOFs that contain input files selected by category from the data set. Different output SOFs are emitted from separate ports, that are connected to data processing actors. For each output port, one, or several, file categories sent to this port are explicitly specified by name. The primary use of the FitsRouter is to select the categories of raw files in a data set that are needed for each data processing actor, whereas products needed as input arrive directly from the data processing actor via dedicated relations.

The routing by category assures that a recipe receives all necessary file categories at the time that it is executed. If there are files with categories that are not needed by the recipes, they can be filtered out by the data processing actor. What remains is the task to select among all of the files of a given category those that should be processed together by the recipe. In Reflex, this is implemented as an actor "SOFCombiner" that bundles the different input files for a recipe into a single SOF. The SOFCombiner has two input ports, one is for mandatory files and another one for optional files. Both of them are multiple ports, i.e. several relations can be connected to either input port. The tokens sent via different relations to a multiple port are in different channels of the port. The SOFCombiner creates a single output SOF that includes input files selected by purpose. The selection rule is that only files with a purpose that is present at each of the input chan$n e l s$, at the mandatory input port, are passed. The desired selection of all files with the appropriate purpose is achieved when at least one of the input channels includes only files that are the necessary input for the recipe, typically the trigger for the recipe. All other channels can include any file, and the SOFCombiner automatically selects the correct input for the recipe. The algorithm used by the SOFCombiner uses comparison of purposes as the only method and consists of the following two steps.

1. Find purposes that are present at each input channel of the mandatory input. A universal purpose counts as a match to any other purpose.

2. Send all files, both from the mandatory and optional port, that match any purpose found in step 1 to the output SOF. Again, a universal purpose counts as a match to any other purpose.

This simple but powerful algorithm assures that the files of the same purpose in the output SOF are necessary and sufficient to run the intended data processing recipe. This fact is then used by a combination of two actors called "SOFSplitter" and "SOFAccumulator". The former splits an input SOF by purpose and emits a separate SOF for each purpose. The latter collects several SOFs that arrive at the same port and combines them into a single SOF. These actors are used in combination with, and always bracket, a data processing actor (see Fig. 6). The net effect of this combination is that the recipes called by the data processing actor are executed multiple times, and the result is a single SOF that includes all of the products. This SOF can then be used by the next SOFCombiner to select the files for the next data processing step.

The algorithms discussed above are an elegant and efficient way to implement the most common routing needs without repetition of information. In addition, explicit operations on file properties can be used to implement special needs. A common application is conditional routing of files, i.e. workflows in which files are routed differently depending on some data properties or user choices. Kepler provides a large number of general purpose actors to implement conditional and/or iterative branches in a workflow, and stand-alone actors, to manipulate the category or purpose of a file, are either provided by Reflex

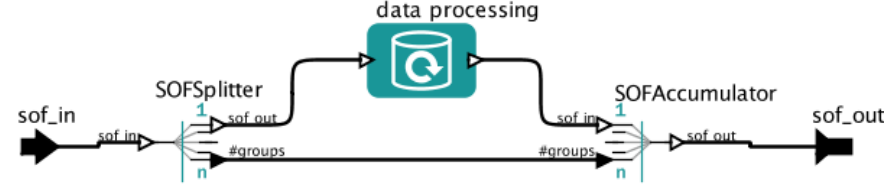

Fig. 6. A data processing actor embedded in a SOFSplitter and SOFAccumulator to manage repetitive executions for files with different purposes.

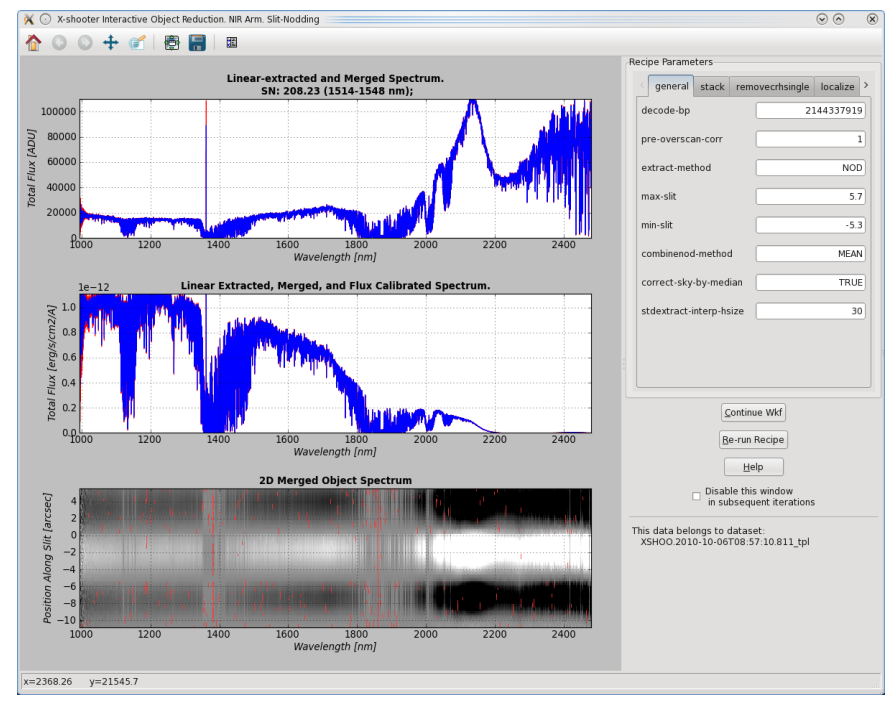

Fig. 7. Example of an interactive interface created with the Reflex Python library. The plots on the left hand side can be interactively manipulated to inspect the data products in different ways. The panel on the right hand side allows the user to modify recipes parameters, and re-execute the recipe or continue with the workflow.

or can easily be implemented (e.g. as a Python script). As emphasized earlier, in any manipulation of the purpose, the purpose should never explicitly be called by name within the workflow to avoid unnecessary dependencies of the workflow on syntax choices in the rules. For example, the case discussed above that a flatfield file is selected to be taken close in time to the science spectrum, but is used to flatfield the flux calibration file can easily be implemented with such a customized purpose processing script.

\subsubsection{Interactive actors}

One reason why automated workflows are an efficient way of data reduction is that the user can intercept the processing at any stage and interact with the workflow. A major contribution to the interactive user experience comes from the workflow application that provides tools to monitor, pause and modify the workflow itself. Additional tools are needed to provide application-specific ways to inspect and influence the execution of the workflow. Reflex provides several interactive actors, and a Python library to implement actors that can create customized plots and allow recipe parameters to be modified during the execution of a workflow. An example of an interface created with this library is shown in Fig. 7. Ready-to-use interactive actors that have been developed for Reflex include the DataSetChooser to interactively inspect and select data sets, the DataFilter to inspect and filter SOFs, and the ProvenanceExplorer to inspect the provenance of a product and its history from repeated runs of a 
workflow. All interactive actors and features can easily be turned off when starting Reflex, to allow a workflow to be run in batch mode once it has been adapted and optimized.

\subsection{Modularity of reflex workflows}

Kepler provides an easy way to create modular workflows. A composite actor is an actor that itself contains a workflow, and composite actors can be nested to arbitrary depth. Placing each data processing actor, together with its supporting actors, into a composite actor leads to a clean and intuitive view of the whole data processing workflow.

The layout of a workflow, whether it is modular or not, does not uniquely define a sequence of actor executions. For example, a scheduler might decide to alternate processing of actors contained in different composite actors. However, workflow execution is more intuitive when each composite actor is completed before processing proceeds to other actors. In Kepler, this can be achieved by placing an appropriately configured director into each composite actor.

\subsection{Book-keeping and product organisation}

The efficiency of the workflow execution relies on rigorous book-keeping that stores relevant information in a database for easy retrieval and processing. During execution of the workflow, the input and output files of each step in the workflow, as well as all parameters used for the processing are stored in the database. For each file, the file name and the checksum are recorded.

The two main uses of the database are the implementation of the lazy mode described in Sect. 2.4, i.e. the keeping track of products for later re-usage, and the organisation of the output files in a user-friendly way. For the lazy mode, checksums and creation dates can be used to detect changes in input files of the same name. The main output of a workflow are the files produced by the final science processing step, i.e. the science data products of a workflow. Intermediate products produced by previous steps are often needed to evaluate the science data products, troubleshoot, or investigate the optimization of the products. For that purpose, each science data product should be associated with the input data and parameters used to generate it. The input files might themselves include products from previous steps, that are associated to the input of that step. At the conclusion of a workflow, all files used and produced during its execution can be organised in a directory tree that can be browsed either with a specialized tool or with a file browser.

\section{Summary and conclusions}

In this paper, we describe how a workflow application can be used to automate an astronomical data processing workflow. We propose a specific design for such a workflow, and present the application Reflex that implements this design within the Kepler workflow engine. The key advantages of automated workflows over alternative methods such as scripting or monolithic data processing programs are the built-in tools for progress monitoring, and the ability to modify the data organisation and data flow efficiently.

The specific advantages of our Reflex implementation include:

1. Selecting and organising the input data is a significant task for any astronomical data reduction. A rule-based data organiser is used to formalize the selection criteria and to fully automate the organisation of data. The automated data organisation can be followed by an interactive step to inspect and modify the chosen data sets.

2. Reflex allows users to monitor the progress of data reduction, interact with the process when necessary, and modify the workflow. A graphical user interface can be used to develop and experiment with workflows. At the same time, workflows can be executed in a completely non-interactive batch mode to allow processing of large data sets and/or for computational time-intensive processing.

3. Re-reduction, after a change in input files or parameters, is efficiently carried out by only re-running those steps that are affected by this change. A modern reduction process might use hundreds of files with dozens of different categories, and any number of data reduction steps. Changing a single parameter in one of the steps or switching a single input file might trigger a complex cascade of necessary re-runs of steps. Recognizing those steps and re-executing them is fully automated in Reflex.

The execution time of the data organiser strongly depends on the complexity of the rules, the total number of files, and the files in each category. For data from a typical observing run, the first time data organization might take on the order of a minute on a typical desktop workstation. In subsequent runs the lazy mode will reduce this time by a very large factor. The execution of the data processing workflow itself adds a fraction of a second to the stand-alone execution of each recipe. The default memory allocation for Reflex is $1536 \mathrm{MB}$ in addition to the memory requirement of the recipes. This allocation can be reduced for simple workflows if necessary.

So far, Reflex workflows have been developed for the most commonly used instruments on ESO's Very Large Telescope (VLT), namely FORS2, SINFONI, UVES, VIMOS, and X-shooter, as well as the newly commissioned KMOS. They are distributed to users to provide them with a pre-packaged workflow that works out-of-the-box to reduce VLT data. All ESO Reflex workflows are intuitive to understand, as each includes a detailed tutorial and a comprehensive demonstration data set. As such, even novice users can easily modify and experiment with the workflows. Reflex workflows are bundled with the corresponding instrument pipelines and the Reflex environment. The whole package can be installed with a single installation script ${ }^{4}$. ESO expects to develop Reflex workflows for all future VLT instruments.

Acknowledgements. The Kepler software is developed and maintained by the cross-project Kepler collaboration, which is led by a team consisting of several of the key institutions that originated the project: UC Davis, UC Santa Barbara, and UC San Diego. We acknowledge useful discussions with Reinhard Hanuschik on some concepts discussed in this paper.

\section{Appendix A: List of Reflex actors}

A complete description of Reflex actors is given by Forchi (2012). Here, we list the standard Reflex actors in alphabetical order.

- DataFilter: interactive actor to inspect and select FITS files.

- DataOrganiser: implementation of the rule-based data organiser as described in text.

- DataSetChooser: interactive actor to inspect files in a data set, edit the selection, and select data sets to be reduced.

- FitsRouter: actor to route files by category.

- IDLActor: interface to configure and execute IDL scripts.

4 http://www. eso.org/reflex/ 
- Is SofEmpty: actor that checks whether an SOF contains files. This actor is used to implement different data flows depending on the availability of some data.

- ObjectToText: actor to present Reflex tokens in human readable form.

- ProductRenamer: actor for renaming FITS files based on keywords of the file.

- ProvenanceExplorer: interactive actor to inspect products produced during a current or previous run of the workflow.

- PythonActor: interface to configure and execute Python scripts.

- RecipeExecuter: interface to configure and execute CPL recipes.

- RecipeLooper: actor to implement looping over one or several recipes.

- SofCreator: actor to create a Reflex Set of Files (SOF) token from a directory with files.

- SopCreator: actor to create Reflex Set of Parameter (SOP) tokens.

- SOFAccumulator: actor to create a single SOF out of several input SOFs that arrive in sequence.

- SOFCombiner: actor to create a single SOF out of several SOFs that are available simultaneously.

- SOFSplitter: actor to split an SOF by file category.

\section{References}

Abhishek, T., \& Sekharb, A. 2007, Comput. Biol. Chem., 31, 305

Altintas, I., Berkley, C., Jaeger, E., et al. 2004, in Scientific and Statistical Database Management, 16th International Conference, 423

Ballester, P., Bramich, D., Forchi, V., et al. 2011, Astronomical Data Analysis Software and Systems XX, 442, 261

Banse, K., Crane, P., Grosbol, P., et al. 1983, The Messenger, 31, 26

Barseghian, et al. 2009, Ecol. Inform, 5, 3

Biretta, J. A., Baggett, S. M., MacKenty, J. W., Ritchie, C. E., \& Sparks, W. B. 1994, in Calibrating Hubble Space Telescope, eds. A. Koratkar, \& C. Leitherer, 8
Cavanagh, B., Jenness, T., Economou, F., \& Currie, M. J. 2008, Astron. Nachr. 329, 295

Curcin, V., \& Ghanem, M. 2008, Biomedical Engineering Conference, CIBEC, Cairo International, 1

Forchi, V. 2012, Reflex User Manual, ESO internal document VLT-MAN-ESO19000-5037

Hook, R. N., Maisala, S., Oittinen, T., et al. 2006, Astronomical Data Analysis Software and Systems XV, 351, 343

Jung, Y., Ballester, P., Banse, K., et al. 2004, Astronomical Data Analysis Software and Systems (ADASS) XIII, 314, 764

Ludäscher, B., Altintas, I., Berkley, C., et al. 2005, Concurrency and Computation: Practice and Experience, 18, 1039

McFarland, J. P., Verdoes-Kleijn, G., Sikkema, G., et al. 2013, Exp. Astron., 35,

McKay, D. J., Ballester, P., Banse, K., et al. 2004, Proc. SPIE, 5493, 444

Nastasi, A., Scodeggio, M., Fassbender, R., et al. 2013, A\&A, 550, A9

Peron, M. 2012, Astronomical Data Analysis Software and Systems XXI, 461, 115

Rose, J., Akella, R., Binegar, S., et al. 1995, Astronomical Data Analysis Software and Systems IV, 77, 429

Schaaff, A., Verdes-Montenegro, L., Ruiz, J. E., \& Santander-Vela, J. 2012, Astronomical Data Analysis Software and Systems XXI, 461, 875

Schmithuesen, O., Erben, T., Trachternach, C., Bomans, D. J., \& Schirmer, M. 2007, Astron. Nachr., 328, 701

Scodeggio, M., Franzetti, P., Garilli, B., et al. 2005, PASP, 117, 1284

Scott, D., Pierfederici, F., Swaters, R. A., Thomas, B., \& Valdes, F. G. 2007 Astronomical Data Analysis Software and Systems XVI, 376, 265

Tody, D. 1993, Astronomical Data Analysis Software and Systems II, 52, 173

Tucker, D. L., Kent, S., Richmond, M. W., et al. 2006, Astron. Nachr., 327, 821

Tsapras, Y., Street, R., Horne, K., et al., 2009, Astron. Nachr., 330, 4

Wells, D. C., Greisen, E. W., \& Harten, R. H. 1981, A\&AS, 44, 363

White, R. L., \& Greenfield, P. 2002, The PyRAF Tutorial, http://stsdas.stsci.edu/stsci_python_sphinxdocs_2.13/docs/ pyraf_tutorial.pdf

Zampieri, S., Chuzel, O., Ferguson, N., et al. 2006, Astronomical Data Analysis Software and Systems XV, 351, 196

Zampieri, S., Forchi, V., 2012, OCA User manual, ESO internal document VLTMAN-ESO-19000-4932.

Zhou, G., 2004 Master Thesis, University of California at Berkeley 